Forum 2018 · 33:139

https://doi.org/10.1007/s12312-018-0402-7

Online publiziert: 20. Februar 2018

(c) Springer Medizin Verlag $\mathrm{GmbH}$, ein Teil von

Springer Nature 2018

HAMBURGER

KREBSGESELLSCHAFT E.V.

Hamburger Krebsgesellschaft e.V.

Hamburg, Deutschland

\title{
Einen Schritt weiter - das Programm für Angehörige
}

zin mit Schwerpunkt Angehörigenforschung, sprach über die vielfältigen Belastungen der Angehörigen und stellte jüngste Forschungsergebnisse aus dem Bereich „Angehörigenforschung“ vor. Sie beantwortete aber auch medizinische Fragen und nahm Stellung zu anderen Sachverhalten wie z. B. der Ernährung von schwer erkrankten Patienten. Fachlich unterstützt wurde die Medizinerin dabei von der pflegerischen Leitung der Palliativstation des Universitätsklinikums Eppendorf, Cornelia Hlawatsch, die mit Kompetenz und viel Erfahrung die sehr konkreten Anliegen der Anwesenden rund um das Thema Versorgung zu beantworten wusste. Monika Bohrmann, Psychologin und Leitung des Beratungsdienstes der Hamburger Krebsgesellschaft e. V., führte durch den Abend und gab Rat bei persönlichen und seelischen Notlagen.

\section{》) Die Belastung der Angehöri- gen ist oft sehr hoch, aber auch sehr individuell}

Gut 20 Interessierte hatten sich in der Beratungsstelle in Hamburg Eppendorf für das Angehörigen-Forum eingefunden. Nachdem die verschiedensten und drängendsten Fragen der Teilnehmer und Teilnehmerinnen geklärt worden waren, ging es über zu einem offenen und oft auch sehr emotionalen Austausch aller Anwesenden. Dabei wurde immer wieder deutlich, wie hoch die Belastung Angehöriger im täglichen Alltag tatsächlich ist und wie individuell sie gebraucht und gefordert werden, da sich die Bedürfnisse der Erkrankten eben auch sehr unterscheiden.

Ab 2018 wird es von der Hamburger Krebsgesellschaft e.V. erstmals auch ein speziell auf Angehörige zugeschnittenes Kurs- und Seminarangebot geben: so finden sich im Kurs- und Veranstaltungsprogramm 2018 u. a. zwei Kunsttherapie Workshops, die ausschließlich für Angehörige von KrebspatientInnen angeboten werden. Hierbei wird es darum gehen nicht nur der Belastung, sondern auch der Entspannung mit kreativ-künstlerischen Mitteln Ausdruck zu verleihen. Wir hoffen, mit unserem Angebot Angehörige noch besser unterstützen zu können.

\section{Korrespondenzadresse}

Monika Bohrmann Leitung Beratungsdienst Hamburger Krebsgesellschaft e.V. Butenfeld 18, 22529 Hamburg, Deutschland info@krebshamburg.de www.krebshamburg.de haberin der maßgeblich von der Ham-
burger Krebsgesellschaft finanzierten Stiftungsprofessur für Palliativmedi- 